\title{
Influence of land-use patterns on benthic diatom communities and water quality in the tropical Monjolinho hydrological basin, São Carlos-SP, Brazil
}

\author{
T Bere* and JG Tundisi \\ Instituto Internacional de Ecologia, Rua Bento Carlos, 750 - Centro, São Carlos - SP - Brasil
}

\begin{abstract}
The objective of this study was to determine the effects of land-use patterns on both diatom community composition and water quality in tropical streams during the dry season. Benthic diatom collections and water quality sampling were done 4 times at 10 sites. A suite of environmental variables that varied with human land-use pattern was assessed to find the combination of variables that best explained patterns of diatom community composition. Canonical Correspondence Analysis (CCA) was used to determine environmental gradients along which species were distributed. A clear relationship between both benthic diatom communities and water quality and land-use pattern was observed with species richness, diversity and evenness, decreasing significantly from the agricultural and forest area to the urban area. Upstream, forested, agricultural sites, with good water quality $\left(\mathrm{BOD}_{5}=0.9\right.$ to $2.6 \mathrm{mg} \cdot \ell^{-1}, \mathrm{DO}=6.8$ to $8.2 \mathrm{mg} \cdot \ell^{-1}$, phosphate $=>2$ to $\left.4.7 \mu \mathrm{g} \cdot \ell^{-1}\right)$, were characterised by Thalassiosira weissflogii, Orthoseira dentroteres, Meridion anceps, Melosira varians, Diatoma spp, Diadesmis contenta, Eunotia papillo, E. bilunaris, E. intermedia, E. sudetica, Aulacoseira alpigena, A. ambigua, Cymbopleura naviculiformis and Stauroneis phoenicenteron. Urban sites, with medium to bad water quality $\left(\mathrm{BOD}_{5}=\sim 7 \mathrm{mg} \cdot \ell^{-1}, \mathrm{DO}=\sim 7\right.$ $\mathrm{mg} \cdot \ell^{-1}$, phosphate $=12.6$ to $\left.83.1 \mu \mathrm{g} \cdot \ell^{-1}\right)$, were characterised by Diadesmis dissimilis, Frustulia rhomboids, Nitzschia scalaris, Nitzschia linearis, Cyclotella pseudostelligera Neidium ampliatum, N. affine, Encyonema silesiacum, E. neomesianum, Aulacoseira granulata, Navicula cryptotenella, Pinnularia legumen, P. gibba, P. divergens, Surirella linearis, S. robusta, and Achnanthidium minutissimum. Downstream urban sites, with very bad water quality (BOD5 $=19.5$ to $26.2 \mathrm{mg} \cdot \ell^{-1}$, DO $=0.4$ to $1.9 \mathrm{mg} \cdot \ell^{-1}$, phosphate $=142.5$ to $\left.248.7 \mu \mathrm{g} \cdot \ell^{-1}\right)$, were characterised by Gomphonema parvulum, G. accuminatum, Nitzschia palea, Nupela praecipua, Sellaphora pupula, Planotidium lanceolata, Fallacia monoculata and Pinnularia subcapitata. Diatom communities demonstrated potential for acting as indicators of changes in water quality due to changes in catchment land-use patterns.
\end{abstract}

Keywords: land-use, benthic diatoms, environmental gradient, agricultural, forests, urban

\section{Introduction}

Land-use - a function of cultural and settlement patterns, economic factors and environmental characteristics (Robbins et al., 1983; Black et al., 1998) - and water resources (hence biotic communities supported by these water resources) are unequivocally linked. For example, results from predominantly agricultural watersheds, with some forests and urban development. showed that land-use had a distinct overall and seasonal effect on water quality (Osborne and Wiley, 1988). Studies in Pinelands of New Jersey also showed substantial land-use effects on natural water quality because of agricultural development, urban densities, and domestic wastewater flow (Zampella, 1994). Nutrient concentration, particularly phosphorus, increases with urban development due to stormwater runoff (Osborne and Willey, 1988; Welch et al., 1998). Increase in nutrient concentration is also a result of other catchment activities, such as runoff from agriculture or sewage treatment plants.

Changes in water quality of lotic systems because of surrounding land-use patterns affects the resulting biotic communities, as the patterns of this biota are responsive to the nature of the prevailing physical and chemical conditions.

\footnotetext{
* To whom all correspondence should be addressed.

푱 0055 (16) 3362 5400; fax: 0055 (16) 3362 5400;

e-mail: taurai@iie.com.br or taubere@yahoo.com

Received 1 March 2010; accepted in revised form 24 November 2010.
}

The integrity of biota inhabiting lotic ecosystems thus provides a direct, holistic and integrated measure of the ecological conditions of a system as a whole (Karr, 1991) and the effects of land-use patterns. The relationship between diatoms and water physical and chemical variables is robust and quantifiable, making diatoms appropriate quantitative indicators of ecological conditions in lotic systems (Pan et al., 1996; Oliveira et al., 2001; Zampella et al., 2007).

Growth and development of benthic diatoms in streams is an outcome of complex interactions between hydrological, chemical and biotic factors. Local factors such as hydrology, light, temperature and water chemistry are controlled by regional factors such as geology, topography or climate, operating at spatial scales of catchments as well as ecoregions (Biggs, 1990). In addition, human land-use activities act to change both local and regional variables at an increasing rate, affecting the resultant biotic communities. Winter and Duthie (2000) showed that the diatom community composition along an urban-rural gradient was correlated with total phosphorus and total nitrogen.

Many studies have related diatom community composition to environmental variation, mainly in the temperate regions (e.g. Biggs, 1990, 1995; Descy and Coste, 1991; Rocha, 1992; Pan et al., 1996; Lobo et al., 1998; Rott et al., 1998; Chessman et al.,1999; Loez and Topalian, 1999; Stevenson and Pan, 1999; Potapova and Charles, 2003, 2005; De la Rey et al., 2008). However, few studies have specifically focused on the effect 

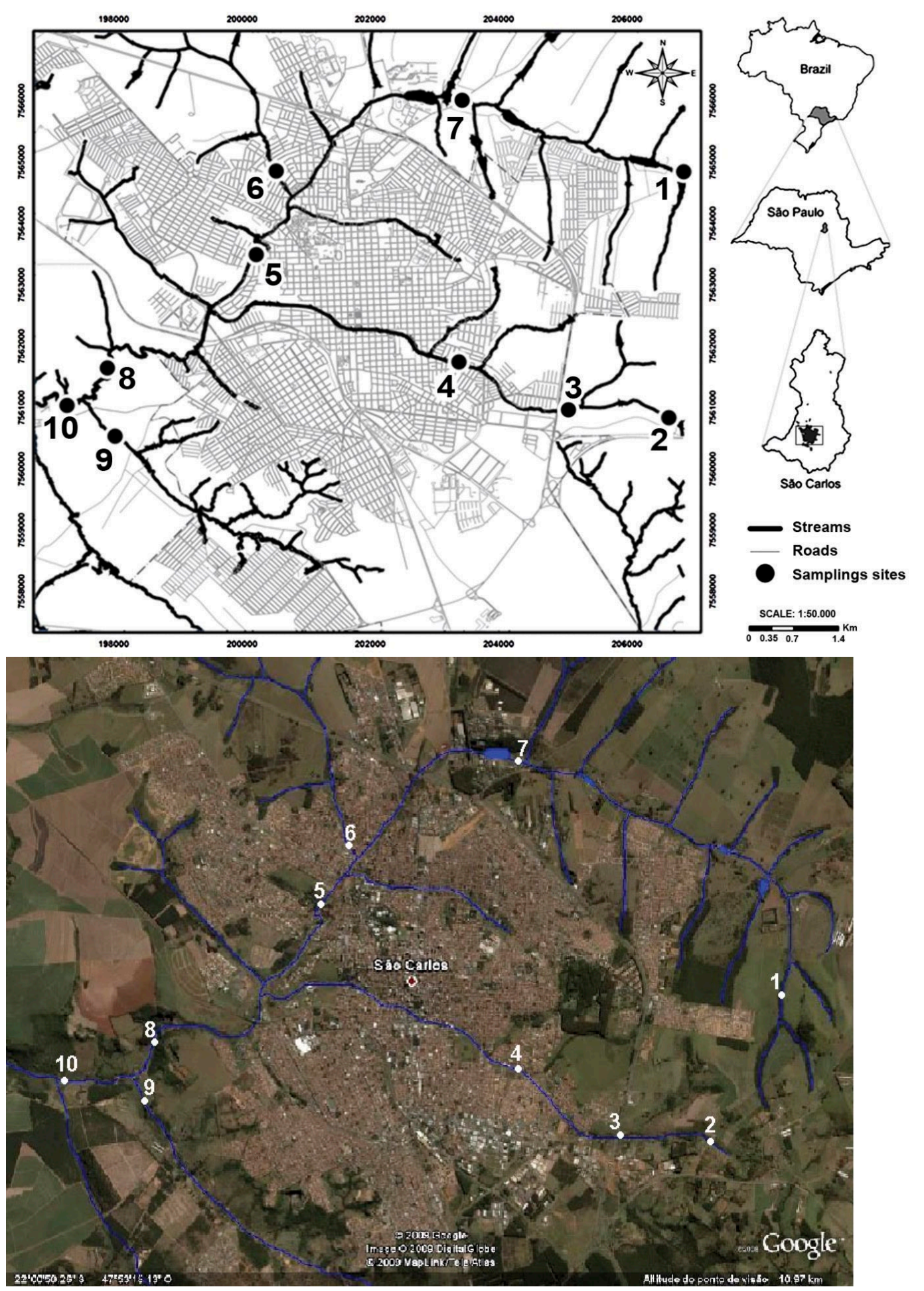

Figure 1

The location of the sampling sites in the study area. (agricultural area)

pastures and farmland preparation for crop cultivation (agricultural area) when outside urban area, buildings and roads (urban area).

of land-use patterns in tropical systems, particularly north of Brazil. Understanding the relationship between land-use patterns, water quality and diatom community composition in streams provides a useful starting point for establishing stream water quality control regulations, conservation goals, ecological restoration efforts, and research hypotheses, all necessary for management of Brazilian and other tropical lotic systems.
The objective of this study was to assess the response of stream diatom assemblages to changes in water quality associated with land-use, i.e., from agricultural and forest areas to urban areas. A suite of environmental variables that varied with human land-use pattern was assessed, in order to find the combination of variables that best explained patterns of diatom community composition. 


\section{Materials and methods}

\section{Study area}

The study area is shown in Fig. 1. Headwaters of the study streams (Monjolinho, Gregório and Água Quente) fall mainly within an agricultural area. Apart from agricultural practices in the headwaters, the streams flow through an urban area of the city of São Carlos, which covers a total area of $1143.9 \mathrm{~km}^{2}$. The area has rugged topography and an average annual temperature of around $19.5^{\circ} \mathrm{C}$, with a mean monthly maximum of $21.9^{\circ} \mathrm{C}$ recorded in January and February and a mean monthly minimum of $15.9^{\circ} \mathrm{C}$ recorded in July.

In 2008, the population of São Carlos was estimated to be 218080 inhabitants (by Instituto Brasileiro de Geografia e Estatística (IBGE)). The current expansion of the city does not meet the technical standards for sewage treatment, garbage collection and urban drainage. Streams in the study area, therefore, receive untreated or semi-treated effluent from various domestic and industrial sources, as well as other diffuse sources, as they flow through the city. The city also expanded without considering environmental, geological and topographical factors, leading to deforestation, erosion, and siltation. This unplanned growth, typical for many Brazilian cities and most developing country cities, results in many problems, including stream health deterioration, loss of primary vegetation, and eutrophication (Dupas et al., 2006; Rörig et al., 2007).

Ten sites were established along Monjolinho River and its tributaries: 3 sites $(1,2$ and 3$)$ in the relatively less impacted agricultural and forested headwaters; 4 sites (4, 5, 6 and 7) in the moderately polluted urban area; and 3 sites $(8,9$ and 10) in the highly polluted area downstream of the urban area (Fig. 1). The rationale in choosing the sampling sites was to cover a pollution gradient of all the stream systems, from relatively unpolluted agricultural headwaters to highly polluted urban downstream sites. Most of the area above upstream sites 1, 2 and 3 was mainly agricultural and characterised by mature deciduous riparian forest strips of about 5 to $50 \mathrm{~m}$ wide, with a mixture of pastures, homesteads and farmland beyond the riparian strip (Table 1). The area also had a low density of buildings, and much of the road network was unpaved. The area above Sites 4, 5, 6, 7, 8, 9 and 10 was mainly urban with more residences along the streams, a mostly paved road network and reduced riparian strips dominated by grass. In some cases, the grass along the stream edges is continuously mown. Some sections of the streams are canalised. Land-use patterns were interpreted from Google Earth Satellite Image System, June 2005 (Fig. 1) using the classification of Anderson et al. (1976) with classes combined to form 3 broad categories (forest, agriculture, and urban).

\section{Water quality}

Water quality sampling was done during the dry season when flow was stable. Four sampling trips were undertaken, two in
September and October 2008 and two in May and June 2009. The dry season was selected to avoid variable effects of the rainy season, such as large variations in water level and velocity, floods and inundations, which affect diatom development, especially growth rate and relative abundance of different species (Duong et al., 2006).

At each site, dissolved oxygen (DO), electrical conductivity, temperature, $\mathrm{pH}$, concentration of total dissolved solids (TDS) and turbidity were measured using a Horiba U-23 and W-23XD Water Quality Meter (Horiba Ltd, Japan). The depth and current velocity were measured at each station with an FP 201 global flow probe (Global Water Instrumentation Inc. Alaska, USA). The percentage of riparian vegetation cover was estimated at each site. The percentage embeddedness (the degree to which large particles are covered with fine particles) was also estimated along each stretch and rated on a $0-5$ scale following Platts et al. (1983). Altitude was determined at each site using a GPS (Northport Systems, Inc. Toronto, Canada). Light intensity was measured using LI-193 Spherical Quantum Sensor (LI-COR Worldwide, Brazil). Water samples for total nitrogen (TN), total phosphorus (TP), biological oxygen demand $\left(\mathrm{BOD}_{5}\right)$, nitrate and phosphate analysis were also collected at each site into acid-cleaned polyethylene bottles following APHA (1988).

In the laboratory, the concentrations of $\mathrm{TN}, \mathrm{TP}, \mathrm{BOD}_{5}$, nitrate and phosphate in the water samples were determined following standard methods (APHA, 1988). $\mathrm{BOD}_{5}$, nitrate, phosphate, $\mathrm{DO}$ (per cent saturation), temperature, $\mathrm{pH}$, turbidity and TDS were used for calculation of a water quality index (WQI), after Brown et al. (1970). This is a method of expressing water quality that offers a simple, stable and reproducible unit of measurement that responds to changes in the principle characteristics of water quality. In this system, the values are given numerical ranking in relation to selected control values for different parameters. The information is then used to compute the standardised distance from control values for each parameter, to produce an index of water quality calculated as the weighted average of variables. The water quality index was calculated; with modification due to absence of faecal coliform data, as follows:

$$
W Q I=\frac{\sum_{i=1}^{n} W i \times Q i}{\sum W i}
$$

where:

$W i$ is the weight

$Q i$ is the quality score of available variable $i$

$W Q I$ is a number between 0 and 100 , with 90 to $100=$ excellent, 70 to $90=$ good, 50 to $70=$ medium, 25 to $50=$ bad and 0 to 25 = very bad (Brown et al., 1970).

\section{Diatom sampling}

Where possible, epilithic (growing on stones), epiphytic (growing on macrophytes), epipelic (growing on mud) and epipsammic (growing on sand) diatoms were sampled separately at each

Table 1
Estimated land-use patterns of catchments above the 10 sampling sites used in the
study


site. Epilithic diatoms were sampled by scraping stones with a toothbrush. Prior to sampling of epilithic surfaces, all substrata were washed with water ejected from a syringe or shaken in the stream to remove any loosely attached sediments and nonepilithic diatoms. At least 5 pebble-to-cobble $(5-15 \mathrm{~cm}$ diameter) sized stones were randomly collected at each sampling site, scraped, and the resulting diatom suspensions pooled to form a single sample, which was then put in a labelled plastic bottle. Epiphytic diatoms were sampled after Fisher and Dunbar (2007). Epipelic and epipsammic diatoms were sampled by pressing a Petri dish lid into the top layer of sand or silt/clay to a depth of 5-7 mm, followed by sliding a spatula blade under the Petri dish to isolate the contents in the dish, which were then stored in a labelled container. Six samples were taken from each station and integrated into a single sample.

In the laboratory, sub-samples of the diatom suspensions were cleaned of organic material using wet combustion with concentrated sulphuric acid and mounted in Naphrax (Northern Biological supplies Ltd. UK. RI = 1.74) following Biggs and Kilroy (2000). Three replicate slides were prepared for each sample. Up to 600 valves per sample (depending on the abundance of diatoms) were identified and counted using the phase contrast light microscope $(1000 \mathrm{X})$. The diatoms were identified to species level based on studies by Metzeltin et al. (2005), Bicudo and Menezes (2006) and Metzeltin and Lange-Bertalot (2007).

\section{Data analysis}

Species diversity $\left(H^{\prime}\right)$, richness $(S)$ and evenness $(E)$ were calculated following Shannon (1949) and were used as measures of community structure. A nonparametric test, Kruskal-Wallis, analogous to analysis of variance, with Mann-Whitney pair-wise comparisons and Bonferroni correlation, was used to compare means of $S, H^{\prime}$ and $E$ among substrates sampled, and among the 4 sampling seasons and 3 sampling site categories. The distributions of turbidity, conductivity, TDS, width, and embeddedness were positively skewed, and therefore were $\ln (X+1)$ transformed (Zar, 1984). Two-way analysis of variance (two-way ANOVA) was used to compare means of environmental variables among the 4 sampling periods and among the 3 site categories.

Multivariate data analyses were performed on the diatom data set to indicate the main gradients of floristic variation and to detect and visualise similarities in diatom samples. Preliminary de-trended correspondence analysis (DCA) was applied to the diatom data set to determine the length of the gradient. This DCA revealed that the gradient was greater than 3 standard deviation units (4.2), justifying the use of unimodial ordination techniques (Ter Braak and Verdonschot, 1995). Thus, Canonical Correspondence Analysis (CCA) was used to investigate relationships between predictor variables and benthic diatom communities from different sites. Preliminary CCA identified collinear variables and selected a subset on inspection of variance inflation factors (VIF< 20; Ter Braak and Verdonschot, 1995). Monte Carlo permutation tests ( 999 unrestricted permutations, $p \leq 0.05$ ) were used to test the significance of the axis and hence determine if the selected environmental variables could explain nearly as much variation in the diatom data as all the measured environmental variables combined. Input for the programme included the relative abundance of diatom taxa that were present in a minimum of 2 samples and had a relative abundance of $\geq 1 \%$ in at least 1 sample. All statistical analyses, DCA, CCA and Kruskal-Wallis, were performed using PAlaeontological STatistics (PAST) software Version 1.90 (Hammer et al., 2009).

\section{Results}

\section{Water quality}

The values of the physical and chemical variables measured are shown in Table 2 . The $\mathrm{pH}$ increased slightly down the

\begin{tabular}{|c|c|c|c|c|c|c|c|c|c|c|}
\hline \multicolumn{11}{|c|}{$\begin{array}{c}\text { Table } 2 \\
\text { The values of physical and chemical variables measured at all of the sites }\end{array}$} \\
\hline Site & 1 & 2 & 3 & 4 & 5 & 6 & 7 & 8 & 9 & 10 \\
\hline Temperature $\left({ }^{\circ} \mathrm{C}\right)$ & $18.3 \pm 1.1$ & $20.9 \pm 1.6$ & $20.6 \pm 1.7$ & $21.2 \pm 1.7$ & $21.2 \pm 1.1$ & $20.4 \pm 1.5$ & $24.0 \pm 2.6$ & $24.8 \pm 3.0$ & $23.0 \pm 1.9$ & $21.3 \pm 2.2$ \\
\hline Conductivity $\left(\mu \mathrm{S} \cdot \mathrm{cm}^{-1}\right)$ & $45.0 \pm 7.5$ & $20.0 \pm 10.5$ & $53.0 \pm 9.5$ & $89.0 \pm 8.9$ & $103.0 \pm 6.4$ & $30.0 \pm 4.0$ & $28.0 \pm 7.7$ & $\begin{array}{l}715.0 \\
\pm 22.3\end{array}$ & $\begin{array}{c}322.0 \\
\pm 191.3\end{array}$ & $\begin{array}{c}283.0 \\
\pm 201.7\end{array}$ \\
\hline $\mathrm{TN}\left(\mathrm{mg} \cdot \ell^{-1}\right)$ & $0.65 \pm 0.3$ & $0.18 \pm 0.3$ & $0.24 \pm 1.4$ & $1.29 \pm 0.4$ & $1.41 \pm 0.5$ & $0.93 \pm 0.7$ & $1.72 \pm 0.5$ & $38.32 \pm 8.3$ & $14.87 \pm 4.1$ & $10.17 \pm 2.9$ \\
\hline $\mathrm{TP}\left(\mathrm{mg} \cdot \ell^{-1}\right)$ & $0.01 \pm 0.0$ & $0.01 \pm 0.0$ & $0.01 \pm 0.1$ & $0.16 \pm 0.2$ & $0.06 \pm 0.1$ & $0.02 \pm 0.0$ & $0.03 \pm 0.0$ & $2.97 \pm 1.0$ & $1.12 \pm 0.2$ & $0.75 \pm 0.2$ \\
\hline Phosphate $\left(\mu \mathrm{g} \cdot \ell^{-1}\right)$ & $<2$ & $<2$ & $4.7 \pm 2.9$ & $83.1 \pm 17.8$ & $12.8 \pm 9.3$ & $<2$ & $<2$ & $19.9 \pm 5.0$ & $\begin{array}{l}142.5 \\
\pm 51.8\end{array}$ & $\begin{array}{r}248.7 \\
\pm 131.2\end{array}$ \\
\hline Nitrate $\left(\mu \mathrm{g} \cdot \ell^{-1}\right)$ & $27.5 \pm 22.3$ & $\mid 175.1 \pm 33.1$ & $524.86 \pm 38.5$ & $964.3 \pm 8.8$ & $\begin{array}{l}1473.6 \\
\pm 232.3 \\
\end{array}$ & $\begin{array}{r}242.5 \\
\pm 667.2 \\
\end{array}$ & $\begin{array}{c}96.7 \\
\pm 595.3 \\
\end{array}$ & \begin{tabular}{c|c|}
$2140.2 \pm 1$ \\
025.4 \\
\end{tabular} & $\begin{array}{c}316.5 \\
\pm 142.5 \\
\end{array}$ & 714.4273 .2 \\
\hline $\mathrm{DO}\left(\mathrm{mg} \cdot \ell^{-1}\right)$ & $7.3 \pm 2.3$ & $8.2 \pm 1.0$ & $7.6 \pm 0.6$ & $6.9 \pm 1.0$ & $7.6 \pm 1.3$ & $7.2 \pm 1.5$ & $6.8 \pm 2.1$ & $1.9 \pm 2.8$ & $2.1 \pm 1.0$ & $0.4 \pm 1.2$ \\
\hline $\mathrm{BOD}_{5}\left(\mathrm{mg} \cdot \ell^{-1}\right)$ & $0.9 \pm 0.2$ & $1.0 \pm 1.2$ & $2.6 \pm 0.2$ & $6.9 \pm 0.2$ & $1.2 \pm 2.3$ & $7.2 \pm 1.1$ & $1.6 \pm 0.6$ & $19.5 \pm 1.1$ & $24.5 \pm 1.2$ & $26.2 \pm 1.3$ \\
\hline $\mathrm{pH}$ & $6.6 \pm 0.8$ & $6.4 \pm 1.0$ & $6.3 \pm 1.0$ & $6.8 \pm 0.9$ & $7.2 \pm 0.4$ & $6.8 \pm 1.0$ & $6.7 \pm 0.6$ & $7.2 \pm 0.5$ & $7.2 \pm 0.4$ & $7.1 \pm 0.4$ \\
\hline Turbidity (NTU) & $5.1 \pm 1.2$ & $4.2 \pm 1.1$ & $4.7 \pm 1.3$ & $19.5 \pm 4.4$ & $11.1 \pm 2.9$ & $13.2 \pm 3.4$ & $7.3 \pm 1.6$ & $45.3 \pm 10.7$ & $53.2 \pm 12.1$ & $60.4 \pm 15.4$ \\
\hline $\operatorname{TDS}\left(\mathrm{g} \cdot \ell^{-1}\right)$ & $29.4 \pm 0.5$ & $13.4 \pm 1.2$ & $22.6 \pm 3.2$ & $57.4 \pm 3.4$ & $66.5 \pm 2.9$ & $19.3 \pm 0.2$ & $18.1 \pm 1.4$ & $\begin{array}{l}457.8 \\
\pm 27.1\end{array}$ & $\begin{array}{l}206.1 \\
\pm 11.1\end{array}$ & $\begin{array}{l}182.0 \\
\pm 34.1\end{array}$ \\
\hline $\begin{array}{l}\text { Light intensity } \\
\left(\mu \mathrm{mol} \cdot \mathrm{s}^{-1} \mathrm{~m}^{-2}\right)\end{array}$ & \begin{tabular}{|l|}
$99.8 \pm 5.3$ \\
\end{tabular} & $347.7 \pm 61.0$ & $439.3 \pm 7.2$ & $\begin{array}{l}612.3 \\
\pm 99.2\end{array}$ & $\begin{array}{l}1806.0 \\
\pm 286.0\end{array}$ & $\begin{array}{c}1757.5 \\
\pm 53.3\end{array}$ & $\begin{array}{c}1357.7 \\
\pm 129\end{array}$ & $\begin{array}{c}2019.8 \\
\pm 61.6\end{array}$ & $\begin{array}{c}1556.3 \\
\pm 88.0\end{array}$ & $\begin{array}{c}2216.8 \\
\pm 19.2\end{array}$ \\
\hline Mean width (m) & $0.8 \pm 0.5$ & $0.8 \pm 0.7$ & $1.4 \pm 1.1$ & $6.0 \pm 3.5$ & $1.0 \pm 0.5$ & $3.5 \pm 2.2$ & $1.6 \pm 0.9$ & $13.0 \pm 1.6$ & $3.7 \pm 3.1$ & $9.0 \pm 2.2$ \\
\hline Mean depth (m) & $0.2 \pm 0.02$ & $0.3 \pm 0.08$ & $0.4 \pm 0.10$ & $0.4 \pm 0.08$ & $0.3 \pm 0.05$ & $0.4 \pm 0.01$ & $0.2 \pm 0.05$ & $0.5 \pm 0.07$ & $0.3 \pm 0.04$ & $0.3 \pm 0.01$ \\
\hline Velocity $\left(\mathrm{m} \cdot \mathrm{s}^{-1}\right)$ & $2.5 \pm 1.3$ & $2.8 \pm 1.4$ & $2.6 \pm 1.3$ & $2.7 \pm 1.2$ & $1.4 \pm 0.6$ & $2.9 \pm 1.1$ & $2.23 \pm 1.1$ & $3.5 \pm 1.8$ & $2.4 \pm 0.9$ & $2.34 \pm 1.0$ \\
\hline Altitude (m) & 761 & 837 & 831 & 794 & 745 & 761 & 774 & 724 & 630 & 627 \\
\hline Canopy cover (\%) & 80 & 95 & 60 & 50 & 4 & 45 & 20 & 20 & 50 & 5 \\
\hline Embeddedness & 0 & 0 & 1 & 3 & 1 & 1 & 2 & 5 & 1 & 4 \\
\hline Stream order & 2 & 1 & 2 & 2 & 3 & 3 & 3 & 3 & 3 & 4 \\
\hline
\end{tabular}




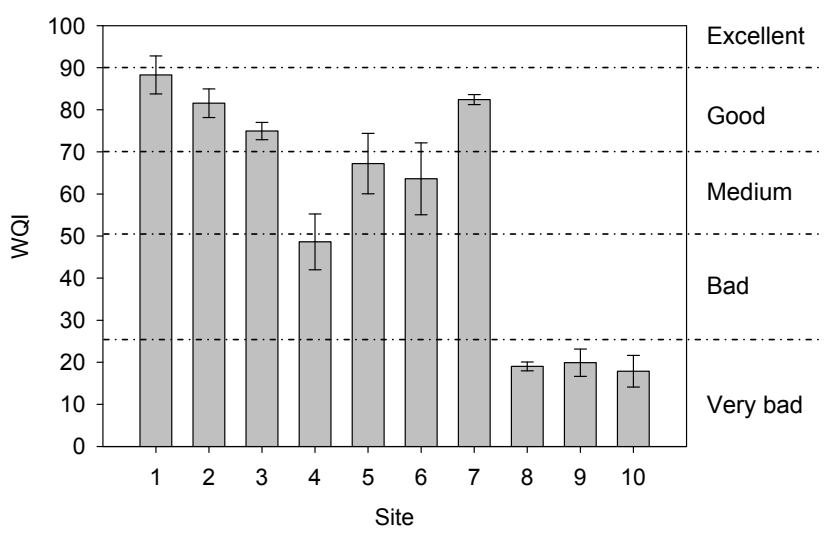

Figure 2

The mean values and standard deviations of water quality index (WQI) recorded at all sites

agricultural to urban gradient, being slightly acidic at upstream sites and slightly alkaline/neutral at downstream sites. However, the difference in $\mathrm{pH}$ among the 3 site categories was not statistically significant (ANOVA, $p>0.05$ ). Temperature increased downstream, but, as in the case of $\mathrm{pH}$, the increase was not significant (ANOVA, $p>0.05$ ). On the other hand, conductivity, $\mathrm{BOD}_{5}$, TDS, turbidity, light intensity, TN, TP, nitrate, phosphate and embeddedness increased significantly downstream (ANOVA, $p<0.05$ ), while DO and percentage riparian vegetation cover decreased significantly downstream (ANOVA, $p<0.05$ ). No significant differences were observed in mean environmental variables among the 4 sampling periods (ANOVA, $p>0.05$ ). This is expected since all sampling was confined to a stable base flow period when variations in water chemistry are low compared to the rainy season. Therefore, the mean environmental variables of the 4 sampling periods at each site were used for subsequent analysis.

The water quality generally tended to deteriorate downstream, as the streams pass through the urban area, due to discharge of treated and untreated domestic and industrial effluent as well as other diffuse sources of pollution from the city (Fig. 2). The mean water quality of Sites 1, 2 and 3, situated in a forested agricultural area, as well as Site 7, was good (with excellent water quality at Site 1 during September and October 2008). Site 4 exhibited bad water quality while Sites 5 and 6 displayed medium water quality. On the other hand, the water quality of Sites 8,9 and 10, situated after the urban area, was very bad.

\section{Community structure}

A total of 208 diatom species belonging to 63 genera were recorded. Twenty-two genera accounted for $91.7 \%$ of the overall diatom community. Of the 208 species observed, 82 species were retained for subsequent analysis (present in a minimum of 2 samples and had a relative abundance of $>1 \%$ in at least 1 sample) (Table 3 , next page).

Species richness, diversity and evenness differed significantly (Kruskal-Wallis, $p<0.05$ ) among sampling sites, tending to be higher in upstream, relatively unpolluted and mainly agricultural and forested area, compared to downstream, polluted, mainly urban sites (Table 4 ). No significant differences (Kruskal-Wallis, $p>0.05$ ) in $S, H^{\prime}$ and $E$ were observed between the substrates sampled for the 4 sampling periods, except for species richness between the September and May and September and June samples (Table 5). Therefore, a pooled data set, consisting of diatoms sampled during 4 sampling periods and among 4 substrates, was used to investigate spatial trends in the composition of diatom communities.

\section{Diatom community - environment relationships}

The results of the CCA are presented in Fig. 3. The first four axes of the species-environment plot accounted for $94.1 \%$ of the total variance in the community due to measured environmental variables (Table 6). Axis 1 and 2 explained $47.7 \%$ and $21.0 \%$, respectively, of the diatom species variance. Monte Carlo unrestricted permutation test indicated that Axis 1 (99 permutations) and Axis 2 (99 permutations of Axis 2 with Axis 1 as a covariable) were statistically significant $(p \leq 0.05)$. $\mathrm{BOD}_{5}$ and $\mathrm{pH}$ were positively associated with the first axis while DO and percentage canopy cover were negatively respectively associated with the first axis. $\mathrm{pH}$ was also positively associated with

\begin{tabular}{|c|c|c|c|c|c|c|c|c|c|c|c|c|}
\hline \multicolumn{13}{|c|}{$\begin{array}{c}\text { Table } 4 \\
\text { The mean species richness }(S) \text {, diversity }\left(H^{\prime}\right) \text { and evenness }(E) \text { for the different } \\
\text { substrates sampled during the study period. }{ }^{*} \text { indicates a missing substrate. }\end{array}$} \\
\hline \multirow{3}{*}{ Site } & \multicolumn{12}{|c|}{ Substrate } \\
\hline & \multicolumn{3}{|c|}{ Silt/clay } & \multicolumn{3}{|c|}{ Sand } & \multicolumn{3}{|c|}{ Stones } & \multicolumn{3}{|c|}{ Vegetation } \\
\hline & $S$ & $H^{\prime}$ & $E$ & $S$ & $H^{\prime}$ & $E$ & $S$ & $\mathbf{H}^{\prime}$ & E & $\mathbf{S}$ & $\mathbf{H}^{\prime}$ & E \\
\hline 1 & 39 & 2.67 & 0.81 & $*$ & $*$ & * & $*$ & $*$ & $*$ & 50 & 2.75 & 0.71 \\
\hline 2 & 36 & 3.28 & 0.92 & $*$ & $*$ & * & 30 & 2.77 & 0.82 & 37 & 3.3 & 0.92 \\
\hline 3 & 41 & 3.24 & 0.88 & 48 & 3.22 & 0.84 & $*$ & * & $*$ & 50 & 3.29 & 0.85 \\
\hline 4 & 19 & 2.54 & 0.86 & 43 & 2.97 & 0.8 & 34 & 3.04 & 0.88 & 28 & 2.93 & 0.9 \\
\hline 5 & 33 & 2.64 & 0.76 & 46 & 3.22 & 0.84 & 30 & 2.8 & 0.84 & 41 & 3.04 & 0.83 \\
\hline 6 & 39 & 2.66 & 0.83 & 28 & 1.91 & 0.89 & 39 & 2.74 & 0.75 & 41 & 2.81 & 0.76 \\
\hline 7 & 67 & 3.35 & 0.8 & 57 & 3.55 & 0.88 & 45 & 3.59 & 0.94 & 65 & 3.4 & 0.81 \\
\hline 8 & 27 & 1.8 & 0.55 & 29 & 2.27 & 0.68 & 20 & 1.65 & 0.56 & 22 & 2.49 & 0.81 \\
\hline 9 & 17 & 2.04 & 0.73 & 20 & 1.93 & 0.64 & 14 & 0.97 & 0.41 & 13 & 1.23 & 0.48 \\
\hline 10 & 36 & 2.61 & 0.73 & 27 & 2.6 & 0.79 & 14 & 0.76 & 0.29 & 23 & 1.48 & 0.45 \\
\hline
\end{tabular}




\begin{tabular}{|c|c|c|c|c|c|c|c|c|c|c|c|}
\hline \multicolumn{12}{|c|}{$\begin{array}{l}\text { Table } 3 \\
\text { The distribution of most abundant diatom species. The sites are arranged in order of increasing } \\
\text { pollution based on water quality index (Figure } 2 \text { ). }+=0-10 \%,++=10-30 \%,+++=>30 \% \text { ). }\end{array}$} \\
\hline Species & Code & 1 & 2 & 7 & 3 & 5 & 6 & 4 & 9 & 8 & 10 \\
\hline Achnanthes exigua Grunow & Aexi & + & + & + & + & + & + & + & & & \\
\hline $\begin{array}{l}\text { Achnanthidium biasolettianum (Grunow) Round \& } \\
\text { Bukhtiyarova }\end{array}$ & Abia & + & + & + & + & + & + & + & & & \\
\hline Achnanthidium minutissimum (Kützing) Czarnecki & Amin & + & + & + & + & + & + & + & & & \\
\hline Amphora copulata (Kützing) Schoeman \& Archibald & Acop & + & + & + & + & + & + & + & & & \\
\hline Aulacoseira agassizii (Hustedt) Simonsen & Aaga & & + & + & + & + & + & + & & & \\
\hline Aulacoseira alpigena (Grunow) Krammer & Aalp & & + & + & + & + & + & + & & & \\
\hline Aulacoseira ambigua (Grunow) Simonsen & Aamb & + & + & + & + & + & + & & & & \\
\hline Aulacoseira distans (Ehrenberg) Simonsen & Adis & & + & + & + & + & + & & & + & \\
\hline Aulacoseira granulata (Ehrenberg) Simonsen & Agra & + & + & + & + & + & + & + & & & \\
\hline Caloneis hyaline Hustedt & Chya & + & + & + & & & & & & & \\
\hline Craticula cuspidata (Kützing) Mann & Ccus & + & & & + & & & & & + & \\
\hline Cyclotella meneghiniana Kützing & Cmen & & + & + & & + & + & + & + & + & + \\
\hline Cyclotella pseudostelligera Hustedt & Cpse & & + & + & + & + & + & & & + & \\
\hline Cyclotella spp. & Cspp & + & + & + & + & + & + & + & + & + & + \\
\hline Cyclotella stelligera (Cleve and Grunow) Van Heurck & Cste & + & & + & & & + & & & & \\
\hline Cymbopleura naviculiformis (Auerswald) Krammer & Cnav & + & + & + & + & + & + & + & & & \\
\hline Diadesmis contenta (Grunow) Mann & Dcon & + & + & + & + & + & + & + & & + & + \\
\hline Diadesmis dissimilis Moser, Lange-Bertalot \& Metzeltin & Ddes & + & & & + & & + & & & & \\
\hline Diatoma spp & Dspp & + & + & + & + & & & & & & \\
\hline Diatoma vulgare Bory & Dvul & + & + & + & + & + & + & + & & & \\
\hline Encyonema neomesianum Krammer & Eneo & + & + & + & + & + & + & + & & & \\
\hline Encyonema silesiacum (Bleisch) Mann & Esil & + & + & + & + & + & + & + & & + & \\
\hline Eunotia bilunaris (Ehrenberg) Mills & Ebil & + & + & + & ++ & + & + & + & & & \\
\hline Eunotia camelus Ehrenberg & Ecam & + & + & + & + & + & + & + & & & \\
\hline Eunotia intermedia (Hustedt) Nörpel and Lange-Bertalot & Eint & ++ & + & + & + & + & + & + & & & \\
\hline Eunotia monodon Ehrenberg & Emon & + & + & + & + & + & + & & & & \\
\hline Eunotia papillo (Ehrenberg) Hustedt & Epop & + & + & + & & + & + & & & & \\
\hline Eunotia pectinalis (Kützing) Rabh & Epec & ++ & + & + & + & + & + & + & & & \\
\hline Eunotia rabenhorstii Cleve \& Grunow & Erab & + & & + & + & & + & & & & \\
\hline Eunotia sudetica Müller & Esud & & + & + & + & + & & + & & & \\
\hline Fallacia monoculata (Hust) Mann & Fmon & & & + & & + & + & + & + & + & + \\
\hline Fragilaria capucina Desmazières & Fcap & + & + & + & + & + & + & + & & & \\
\hline Fragilaria intermedia Grunow & Fint & + & + & + & + & + & + & + & & & + \\
\hline Frustulia rhomboides (Rabenhorst) De Toni & Frho & + & + & + & + & + & ++ & + & & & \\
\hline Frustulia saxonica Rabenhorst & Fsax & + & + & + & + & + & + & + & & & \\
\hline Frustulia vulgaris (Twaithes) de Toni & Fvul & + & + & + & + & + & + & + & & & \\
\hline Gomphonema accuminatum Ehrenberg & Gacc & + & + & + & + & + & + & + & & + & \\
\hline Gomphonema angustatum (Kützing) Rabenhorst & Gang & + & + & + & + & + & + & + & + & & + \\
\hline Gomphonema augur (Ehrenberg) Lange-Bertalot & Gaug & + & + & + & + & + & + & + & & & \\
\hline Gomphonema gracile Ehrenberg & Ggra & + & + & + & + & + & + & + & & & \\
\hline Gomphonema olivaceum (Hornemann) Brébisson & Goli & + & + & + & + & + & + & + & & & \\
\hline Gomphonema parvulum (Kützing) Kützing & Gpar & + & + & + & + & + & + & + & ++ & + & + \\
\hline Gomphonema turris Ehrenberg & Gtur & + & + & + & & + & & & & & \\
\hline Hantzschia amphioxys (Ehrenberg) Grunow & Hamp & + & + & + & + & + & + & + & & & \\
\hline Luticola goeppertiana (Bleisch) Mann & Lgeo & + & + & + & + & + & + & + & + & + & ++ \\
\hline Melosira varians Agardh & Mvar & & + & + & + & + & + & + & & & \\
\hline Meridion anceps (Ehrenberg) Williams & Manc & + & + & + & + & + & & + & & & \\
\hline Navicula cryptocephala (Grunow) Cleve & Nery & + & + & + & + & + & + & + & & & + \\
\hline Navicula cryptotenella Lange-Bertalot & Ncrt & + & + & + & + & + & + & + & & + & \\
\hline Navicula oblonga Kützing & Nobl & + & + & + & + & + & + & + & & & \\
\hline Navicula radiosa Kützing & Nrad & + & + & + & + & + & + & + & & & \\
\hline Navicula rostellata Kützing & Nros & & & + & + & + & + & + & & & \\
\hline Neidium affine (Ehrenberg) Pfitzer & Naff & + & + & + & + & + & + & + & & & \\
\hline Neidium ampliatum (Ehrenberg) Krammer & Namp & & & + & & & + & & & & \\
\hline Nitzschia linearis (Agardh) Smith & Nlin & + & + & + & + & + & + & + & + & & \\
\hline Nitzschia palea (Kützing) Smith & Npal & & + & + & + & + & ++ & ++ & ++ & +++ & +++ \\
\hline Nitzschia recta Hantzsch ex Rabenhorst & Nrec & + & + & + & + & + & + & + & + & + & + \\
\hline Nitzschia scalaris (Kützing) Grunow & Nsca & + & & + & + & + & + & + & & & \\
\hline Nupela praecipua (Reichardt) Reichardt & Npra & + & + & + & + & ++ & + & + & ++ & + & + \\
\hline Orthoseira dentroteres (Ehrenberg) Crawford & Oden & + & + & & + & & & & & & \\
\hline Pinnularia braunii (Grunow) Cleve & Pbra & + & & + & + & + & + & + & + & + & + \\
\hline Pinnularia divergens $\mathrm{Krammer}$ & Pdiv & + & & + & + & + & + & + & & & \\
\hline Pinnularia gibba Ehrenberg & Pgib & + & + & + & + & + & + & + & + & + & + \\
\hline Pinnularia lata (Brébisson) Rabenhorst & Plat & + & & + & + & + & + & + & & & \\
\hline Pinnularia legumen Ehrenberg & Pleg & & & + & + & & + & + & & & \\
\hline Pinnularia microstauron (Ehrenberg) Cleve & Pmic & + & & + & & + & + & + & & + & \\
\hline Pinnularia subcapitata Gregory & Psub & & & + & & + & + & + & + & + & + \\
\hline
\end{tabular}




\begin{tabular}{|l|l|l|l|l|l|c|c|c|c|c|c|}
\hline Placoneis clementis (Grunow) Cox & Pcle & & + & + & + & + & + & + & & & \\
\hline Planothidium dubium (Grunow) Round and Bukhtiyarova & Pdub & + & + & + & + & + & + & + & & & \\
\hline Planothidium lanceolatum (Brébisson) Grunow & Plan & + & + & + & + & + & + & + & + & + & + \\
\hline Planotidium heteroideum & Phet & & + & + & + & + & + & + & & & \\
\hline Pleurosigma compactum Greville & Pcom & & & + & & & + & + & & & \\
\hline Psammothidium subatomoides (Hustedt) Bukhtiyarova & Psuba & + & + & + & + & + & + & + & + & & + \\
\hline Rhoicosphenia abbreviata (Agardh) Lange-Bertalot & Rabb & + & + & + & + & + & + & + & + & + & + \\
\hline Sellaphora pupula (Kützing) Mereschkowsky & Spup & + & + & + & + & + & + & + & + & + & + \\
\hline Stauroneis phoenicenteron (Nitzsch) Ehrenberg & Spho & + & + & + & + & + & + & + & & & \\
\hline Surirella angusta Kützing & Sang & + & & + & + & + & + & + & & + & \\
\hline Surirella linearis Smith & Slin & + & + & + & + & + & + & + & & & \\
\hline Surirella ovata Kutzing & Sova & + & & & + & + & + & & & & \\
\hline Surirella robusta Enrenberg & Srob & + & & + & + & & + & + & & \\
\hline Thalassiosira weissflogii (Grunow) Fryxell and Hasle & Twei & + & + & & + & & & & & & + \\
\hline Ulnaria ulna Nitzsch) Compère & Uuln & + & + & + & + & + & + & + & & + & + \\
\hline
\end{tabular}

\begin{tabular}{|c|c|c|c|c|c|c|c|c|c|c|c|}
\hline \multicolumn{12}{|c|}{$\begin{array}{l}\text { Table } 5 \\
\text { Results of Kruskal-Wallis with Mann-Whitney pairwise comparisons of species richness }(S), \\
\text { Shannon's diversity }\left(H^{\prime}\right) \text { and evenness }(E) \text { indices during the } 4 \text { sampling periods (s = September } \\
2008,0=\text { October } 2008, \mathrm{~m}=\text { May } 2009, \mathrm{j}=\text { June 2009). Significant differences are highlighted. }\end{array}$} \\
\hline & S-s & $H^{\prime}-\mathbf{s}$ & E-s & S-O & $H^{\prime}-0$ & E-o & S-m & $H^{\prime}-\mathbf{m}$ & E-m & $S-\mathbf{j}$ & $H^{\prime}-\mathrm{j}$ \\
\hline$S$-S & & & & 0.15 & & & 0.00 & & & 0.03 & \\
\hline$H^{\prime}-\mathrm{s}$ & & & & & 0.24 & & & 0.17 & & & 0.58 \\
\hline$E$-s & & & & & & 0.95 & & & 0.10 & & \\
\hline$S$-n & 1 & & & & & & 0.16 & & & 0.44 & \\
\hline$H^{\prime}-\mathrm{n}$ & & 1 & & & & & & 0.74 & & & \begin{tabular}{|l}
0.78 \\
\end{tabular} \\
\hline$E$-n & & & 1 & & & & & & 0.08 & & \\
\hline$S$-m & 0.2 & & & 1 & & & & & & \begin{tabular}{|l|}
0.79 \\
\end{tabular} & \\
\hline$H^{\prime}-\mathrm{m}$ & & 1 & & & 1 & & & & & & 0.66 \\
\hline$E$-m & & & 1 & & & 1 & & & & & \\
\hline$S-\mathrm{j}$ & 1 & & & 1 & & & 1 & & & & \\
\hline$H^{\prime}-\mathrm{j}$ & & 1 & & & 1 & & & 1 & & & \\
\hline$E-\mathrm{j}$ & & & 1 & & & 1 & & & 1 & & \\
\hline
\end{tabular}

the second axis while stream velocity was negatively associated with the second axis.

CCA Axis 1 and 2 separated the sites into 3 groups along an agricultural to urban gradient (human-induced increase in nutrients and organic pollution and decrease in DO and percentage canopy cover). The first group consisted of forested and agricultural sites with good mean water quality, Sites 1, 2 and 3 that were negatively associated with the first and second axis in the bottom left quadrant (Fig. 3). These sites were associated with high canopy cover (which was negatively correlated to temperature, light intensity and mean stream width) and low $\mathrm{BOD}_{5}$ (which was positively correlated with TDS, TN, TP, nitrate, phosphate, conductivity and depth). Diatom species characterising these sites include species such as T. weissflogii, $O$. dentroteres, M. anceps, M. varians, Diatoma spp, D. contenta, E. papillo, E. bilunaris, E. intermedia, E. sudetica, A. alpigena, A. ambigua, C. naviculiformis and S. phoenicenteron.

The second group consisted of bad to medium water quality urban sites, Sites 4,5 and 6 , that were negatively and positively associated with the first and second axis, respectively, in the upper left hand quadrant. Site 7 was also placed in this group despite its good water quality. Diatom species characterising these sites include species such as $D$. dissimilis, F. rhomboids, S. robusta, N. scalaris, C. pseudostelligera N. ampliatum, E. silesiacum, E. neomesianum, $N$. affine, $N$. linearis, $A$. granulata, N. cryptotenella, $P$. legumen, $P$. gibba, $P$. divergens, $S$. linearis and A. minutissimum. The third group consisted of very bad water quality downstream, Sites 8,9 and 10 , positively associated with the first axis. These sites were associated

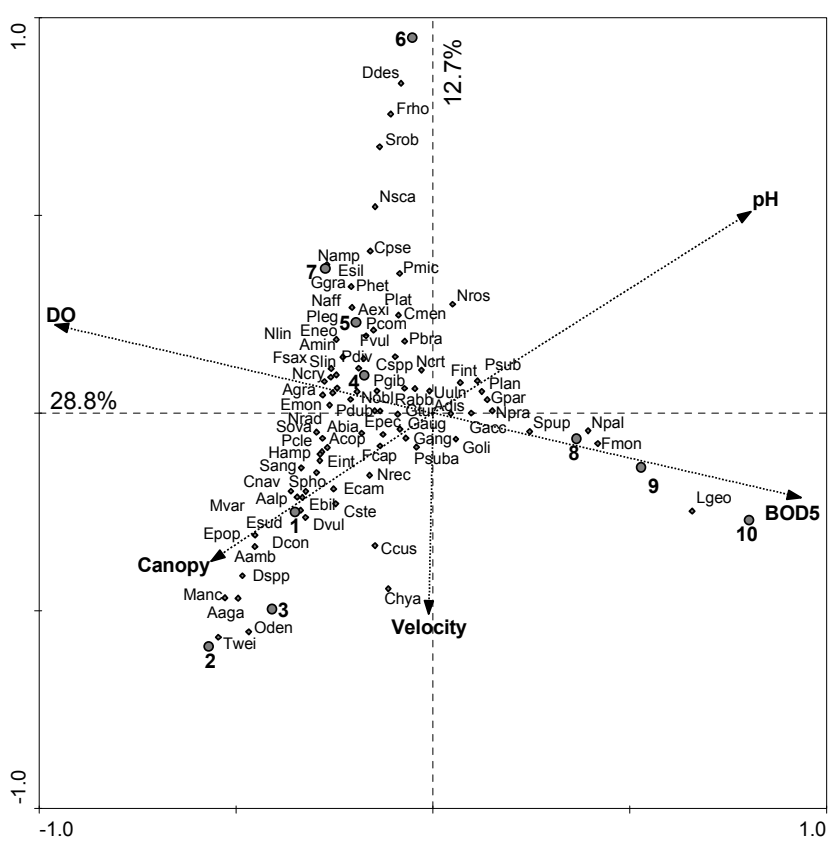

Figure 3

Ordination diagram based on canonical correspondence analysis (CCA) of most dominant diatom species composition in 10 sampling sites with respect to 5 environmental variables (percentage canopy cover, BOD5, stream velocity, $\mathrm{pH}$ and $\mathrm{DO}$ ). Taxa codes correspond to those in Table 3. 


\begin{tabular}{|c|c|c|c|c|}
\hline \multicolumn{5}{|c|}{$\begin{array}{c}\text { Table } 6 \\
\text { Summary of the canonical correspondence analysis (CCA) of most dominant } \\
\text { diatom species composition in } 10 \text { sampling sites with respect to } 5 \text { environmental } \\
\text { variables (percentage canopy cover, BOD5, stream velocity, pH and DO) }\end{array}$} \\
\hline Axes & 1 & 2 & 3 & 4 \\
\hline Eigenvalues & 0.41 & 0.18 & 0.11 & 0.11 \\
\hline Species-environment correlations & 0.97 & 0.99 & 0.97 & 0.94 \\
\hline Cumulative percentage variance of & & & & \\
\hline Species data & 28.8 & 41.4 & 49.3 & 56.8 \\
\hline Species-environment relation & 47.7 & 68.7 & 81.7 & 94.1 \\
\hline
\end{tabular}

with high nutrients and organic pollution, low percentage canopy cover and low DO. These sites were associated with such species as N. palea, G. parvulum, S. pupula,

N. praecipua, P. lanceolata, F. monoculata, P. subcapitata and G. accuminatum. These species have been reported to be pollution tolerant (Biggs and Kilroy, 2000; Potapova and Charles, 2003; Duong et al., 2006).

\section{Discussion}

Diatom community structure showed a clear relationship with changes in water quality associated with changes in land-use pattern. For instance, species richness, diversity and evenness decreased along a forest and agricultural to urban land-use gradient (human-induced increasing nutrient and organic pollution gradient). Low pollution tolerant species, such as T. weissflogii, $O$. dentroteres, $M$. anceps, $M$. varians, Diatoma spp,

D. contenta, E. papilla, E. bilunaris, E. intermedia, E. sudetica, A. alpigena, A. ambigua, C. naviculiformis and S. phoenicenteron were replaced by moderate pollution tolerant species such as D. dissimilis, F. rhomboids, S. robusta, N. scalaris, C. pseudostelligera N. ampliatum, E. silesiacum, E. neomesianum, $N$. affine, $N$. linearis, A. granulata, $N$. cryptotenella, P. legumen, $P$. gibba, P. divergens, S. linearis and A. minutissimum. The latter group of species was replaced by high pollution tolerant species such as N. palea, G. parvulum, S. pupula, $N$. praecipua, P. lanceolata, F. monoculata, P. subcapitata and G. accuminatu, which are known to be resistant to organic and high ionic strength and conductivity (Van Dam et al., 1994; Biggs and Kilroy, 2000; Potapova and Charles, 2003). These species have also frequently been recorded in waters that are nutrient rich and poorly oxygenated, with high electrical conductivity (Van Dam et al., 1994; Bere and Tundisi, 2009).

Researchers have reported different effects of land-use patterns on benthic diatom community structure, in terms of diversity and evenness. Cuffney et al. (1997) found that taxa richness of benthic-algal communities, with diatoms as a dominant component, did not show a strong relationship with land-use or water quality. Hill et al. (2001) concluded that diatom community structure has no predictable relationship with human-related watershed disturbances. However, Chessman et al. (1999) associated an increase in genus richness with increasing alkalinity, conductivity, hardness, and $\mathrm{pH}$ related to land-use pattern, and Hill et al. (2003) reported that diatom-species richness was positively correlated with total phosphorus and nitrogen also related to land-use pattern. This supports the results of the current study. Several other studies have also demonstrated the association of land-use with diatom community structure, in support of the present study (e.g. Morgan, 1987; Pan et al., 1996; Kutka and Richards, 1996; Leland and Porter, 2000; Carpenter and Waite, 2000; Salomoni et al., 2006).
Perhaps the study most relevant to the current study is that conducted by Gómez and Licursi (2001) on epipelic diatoms in the tropical streams of Argentina, in an environment similar to this study. They classified 88 diatom species frequently found in the epipelion of Pampean streams and rivers, based on ecological preferences according to water quality. Thirteen of these species were among the most frequently occurring species in our study (Table 3): A. minutisimum, A. copulata, E. silesiacum, F. capucina, G. angustatum, G. parvulum, H. amphioxys, $N$. cryptocephala, $N$. linearis, $N$. palea, N. recta, S. phoenicenteron, $P$. gibba and S. pupula. The distribution of all these species in relation to pollution was consistent with that described by Gómez and Licursi (2001).

The CCA results showed that $\mathrm{BOD}_{5}$ (also positively correlated with TDS, TP, TN, phosphate, nitrate, conductivity, turbidity, temperature and embeddedness) was important in structuring benthic diatom communities in the study area. Environmental monitoring studies in Southern Brazil (e.g. Oliveira et al., 2001; Lobo et al. 2002, 2004; Salomoni et al., 2006) showed that diatom communities in lotic ecosystems are a result of the interaction of variables characterising the process of organic contamination as well as eutrophication. In this study, highly eutrophic and high organic pollution sites, Sites 8, 9 and 10, had high relative abundances of such species as G. angustatum, G. parvulum, N. palea, N. praecipua and S. pupula. N. palea, G. parvulum, S. pupula, N. praecipua, P. lanceolata, F. monoculata, P. subcapitata and G. accuminatum. These species have been classified as typical for eutrophic and organically polluted environments (Kelly and Whitton, 1995; Van Dam et al., 1994). Numerous studies conducted on lotic benthic diatoms sampled in various countries have shown the influence of organic pollution (Sládecék, 1986; Salomoni et al., 2006), eutrophication (Kelly and Whitton, 1995; Ponader et al., 2007; Lavoie et al., 2008), and dissolved oxygen (Biggs and Kilroy, 2000; Potapova and Charles, 2003) in structuring of diatom communities.

Canopy cover (which was negatively correlated to temperature, light intensity, mean stream width and turbidity and is highly dependent on land-use patters) was also found to be important in structuring benthic diatom communities in the study area. This is because of the importance of light for diatom photosynthesis (Ter Braak and Van Dam, 1989; Biggs, 1990, 1995; Patrick and Hendrickson, 1993; Pan et al., 1996; Biggs and Kilroy, 2000; Carpenter and Waite 2000; Potapova and Charles, 2002, 2003, 2005). Diatom communities in forested agricultural sites were, thus, different from those from open urban sites.

However, stream velocity (not directly related to land-use pattern) was also found to be important in structuring diatom communities in the study area. Site 7 was grouped together with bad to medium water quality sites, Sites 4, 5 and 6, because of its low velocity, despite its good water quality. The 
importance of velocity in structuring benthic diatom communities has also been reported by other researchers (e.g. Biggs, 1990, 1995; Patrick and Hendrickson, 1993; Pan et al., 1996; Biggs and Kilroy, 2000; Potapova and Charles, 2005).

\section{Acknowledgements}

This study was made possible by the provision of funds from Third World Academy of Science (TWAS) through Conselho Nacional de Desenvolvimento Científico e Tecnológico (CNPq). I also wish to thank the Instituto Internacional de Ecologia management and staff for their support during the course of this study.

\section{References}

ANDERSON JR, HARDY EE, ROACH JT and WITMER RE (1976) A Land Use and Land Cover Classification System For Use With Remote Sensor Data. Geological Survey Professional Paper No. 964, U.S. Government Printing Office, Washington, DC. 28.

APHA (1988) Standard Methods for the Examination of Water and Waste Water (20th edn.). American Public Health association, Washington, D.C.

BERE T and TUNDISI JG (2009) Weighted average regression and calibration of conductivity and $\mathrm{pH}$ of benthic diatoms in streams influenced by urban pollution - Sao Carlos/SP Brazil. Acta Limnol. Brasil. 21 317-325.

BICUDO CEM and MENEZES M (2006) Gêneros de água de águas continentais do Brazil: chave para identificação e descrições. Rima Editora, São Carlos-SP, Brazil.

BIGGS BJF (1990) Periphyton communities and their environmental gradients in New Zealand Rivers. New Zeal. J. Freshwater Res. 24 367-386.

BIGGS BJF (1995) The contribution of flood disturbances, catchment geology and land-use to the habitat template of periphyton in stream ecosystems. Freshwater Biol. 33 419-438.

BIGGS BJF and KILROY C (2000) Stream Periphyton Monitoring Manual. NIWA, Christchurch, New Zealand.

BLACK AE, STRAND E, WRIGHT RG, SCOTT JM, MORGAN P and WATSON C (1998) Land-use history at multiple scales: implications for conservation planning. Landsc. Urb. Plann. 43 49-63.

BROWN RM, McLELLAND NJ, DEININGER RA and TOZER RG (1970) A Water Quality Index Do We Dare? Water Sewage Works October 339-343.

CARPENTER KD and WAITE IR (2000) Relations of habitatspecific algal assemblages to land-use and water chemistry in the Willamette Basin, Oregon. Environ. Monit. Assess. 64 247-257.

CHESSMAN B, GROWNS I, CURREY J and PLUNKETT-COLE N (1999) Predicting diatom communities at the genus level for the rapid biological assessment of rivers. Freshwater Biol. 41 317-331.

CUFFNEY TF, MEADOR MR, PORTER SD and GURTZ MF (1997) Distribution of Fish, Benthic Invertebrate, and Algal Communities in Relation to Physical and Chemical Conditions, Yakima River Basin, Washington, 1990, US. Geological Survey Water Resources Investigations Report 96-4280, Raleigh, North Carolina.

DE LA REY PA, ROUX H, VAN RENSBURG L and VOSLOO A (2008) On the use of diatom-based biological monitoring Part 2: A comparison of the response of SASS 5 and diatom indices to water quality and habitat variation. Water $S A 34$ 61-69.

DESCY JP and COSTE M (1991) A test of methods for assessing water quality based on diatoms. Verhandlungen Internat. Verhandlungen Internat. Verein. Theoret. Angewandte Limnol. 24 2112-2116.

DUONG T, COSTE M, FEURTET-MAZEL A, DANG D, GOLD C, PARK Y and BOUDOU A (2006) Impact of Urban Pollution from the Hanoi Area on Benthic Diatom Communities Collected from the Red, Nhue and Tolich Rivers (Vietnam). Hydrobiol. 563 201-216.

DUPAS FA, SILVA E SOUZA AT, MATSUMURA-TUNDISI T, TUNDISI JG and RÖHM SA (2006) Environmental indicators in the planning and management of watersheds. In: Tundis
JG, Matsumura-Tundisi T and Galli CS (Eds.) Eutrophication in South America: Causes, Consequences and Technologies for Management and Control. International Institute of Ecology, São Carlos-SP, Brazil.

FISHER J and DUNBAR MJ (2007) Towards a representative periphytic diatom sample. Hydrol. Earth Syst. 11 399-407.

GÓMEZ N and LICURSI M (2001) The Pampean Diatom Index (IDP) for assessment of rivers and streams in Argentina. Aquat. Ecol. 35 173-181.

HAMMER O, HARPER DAT and RYAN PD (2009) PAST - PAlaeontological STatistics, version 1.90. URL: http://folk.uio.no/ohammer/ past (Accessed DATE).

HILL BH, HERLIHY AT, KAUFMANN PR, DECELLES SJ and VANDER BORGH MA (2003) Assessment of streams of the eastern United States using a periphyton index of biotic integrity. Ecol. Indic. 2 325-338

HILL BH, STEVENSON RJ, PAN Y, HERLIHY AT, KAUFMANN PR and JOHNSON CB (2001) Comparison of correlations between environmental characteristics and stream diatom assemblages characterized at genus and species level. J. N. Am. Benthol. Soc. 20 299-310.

KARR JR (1991) Biological integrity: a long-neglected aspect of water resource management. Ecol. Appl. 1 66-84.

KELLY MG and WHITTON BA (1995) The trophic diatom index: a new index for monitoring eutrophication in rivers. J. Appl. Phycol. $7433-444$.

KUTKA FJ and RICHARDS C (1996) Relating diatom assemblage structure to stream habitat quality. J. N. Am. Benthol. Soc. 15 469-480.

LAVOIE I, CAMPEAU S, DARCHAMBEAU F, CABANA G and DILLON PJ (2008) Are diatoms good integrators of temporary variability in stream water quality? Freshwater Biol. 53 827-841.

LELAND HV and PORTER SD (2000) Distribution of benthic algae in the upper Illinois River basin in relation to geology and land-use. Freshwater Biol. 44 279-301.

LOBO EA, CALLEGARO VL and BENDER P (2002) Utilização de algas diatomáceas epilíticas como indicadoras da qualidade da água em rios e arroios da Região Hidrográfica do Guaíba. RS, EDUNISC, Santa Cruz do Sul, Brasil.

LOBO EA, CALLEGARO VL, WETZEL CE, HERMANY G and BES D (2004) Water quality study of Condor and Capivara streams, Porto Alegre municipal district, RS, Brazil, using epilithic diatoms biocenoses as bioindicators. Oceanol. Hydrobiol. Stud. 33 77-93.

LOBO EA, CALLEGARO VLM, BENDER EP and ASAI K (1998) Water quality assessment of rivers of Southern Brazil using epilithic diatom assemblages. 15th Int. Diatom Symp. 28 September - 2 October 1998, Perth, Australia.

LOEZ CR and TOPALIAN ML (1999) Use of algae for monitoring rivers in Argentina with a special emphasis for Reconquista River (region of Buenos Aires). In: Prygiel J, Whitton BA, Bukowska $\mathrm{J}$ (eds) Use of Algae for Monitoring Rivers III. Agence de l'Eau Artois-Picardie, Douai, p. 72-83.

METZELTIN D and LANGE-BERTALOT H (2007) Tropical Diatoms of South America II. Iconographia Diatomologica 18 1-877.

METZELTIN D, LANGE-BERTALOT H and GARCÍA-RODRÍGUEZ F (2005) Diatoms of Uruguay. Iconographia Diatomologica 15 1-736 1-736.

MORGAN MD (1987) Impact of nutrient enrichment and alkalinisation on periphyton communities in the New Jersey Pine Barrens. Hydrobiologia 144 233-241

OLIVEIRA MA, TORGAN LC, LOBO EA and SCHARZBOLD A (2001) Association of periphytic diatom species of artificial substrate in lotic environments in the Arroio Sampaio basin, RS, Brazil: relationships with abiotic variables. Braz. J. Biol. 6 523-540.

OSBORNE LL and WILEY MJ (1988) Empirical relationships between land-use/cover and stream water quality in agricultural watershed. J. Environ. Manage. 26 9-27

PAN Y, STEVENSON RJ, HILL BH, HERLIHY AT and COLLINS GB (1996) Using diatoms as indicators of ecological conditions in lotic systems: a regional assessment. J. N. Am. Benthol. Soc. 15 481-495. 
PATRICK R and HENDRICKSON J (1993) Factors to consider in interpreting diatom changes. Nova Hedwigia Beihelf 106 361-377.

PLATTS WS, MEGAHAN WF and MINSHALL WG (1983) Methods for evaluating stream, riparian, and biotic conditions. General Technical Report INT-138, USDA Forest Service, Rocky Mountain Research Station. Ogden, UT.

PONADER KC, CHARLES DF and BELTON TJ (2007) Diatom-based $\mathrm{TP}$ and TN inference models and indices for monitoring nutrient enrichment of New Jersey streams. Ecol. Indic. 7 79-93.

POTAPOVA M and CHARLES DF (2002) Benthic diatoms in USA Rivers: distributions along speciation and environmental gradients. J. Biogeogr. 29 67-187.

POTAPOVA M and CHARLES DF (2003) Distribution of benthic diatoms in U.S. rivers in relation to conductivity and ionic composition. Freshwater Biol. 48 1311-1328.

POTAPOVA M and CHARLES DF (2005) Choice of substrate in algae-based water quality assessment. J. N. Am. Benthol. Soc. 24 415-427.

ROBBINS WG, FRANK RJ and ROSS RE (1983) Regionalism and the Pacific Northwest. Oregon State University Press, Corvallis.

ROCHA AA (1992) Algae as indicators of water pollution.I In: Cordeiro-Marino M, Azevedo MTP, Santána CL, Tomita NY, Pastino EM (eds.) Algae and Environment: A General Approach. Sociedade Brasileira de Ficologia, CETESB, São Paulo. 34-55.

RÖRIG LR, TUNDISI JG, SCHETTINI CAF, PEREIRA-FILHO J, MENEZES JT, ALMEIDA TCM, URBAN SR, RADETSKI CM, SPERB RC, STRAMOSK CA, MACEDO RS, CASTRO-SILVA MA and PEREZ JAA (2007) From a water resource to a point pollution source: the daily journey of a coastal urban stream. Braz. J. Biol. 67 597-609.

ROTT E, DUTHIE HC and PIPP E (1998) Monitoring organic pollution and eutrophication in the Grand River, Ontario, by means of diatoms. Can. J. Fish. Aquat. Sci. 55 1443-1453.
SALOMONI SE, ROCHA O, CALLEGARO VL and LOBO EA (2006) Eplithic diatoms as indicators of water quality in the Gravataí River, Rio Grande do Sul, Brazil. Hydrobiologia 559 233-246.

SHANNON CE (1949) The Mathematical Theory of Communication. University of Illinois Press, Urbana.

SLÁDECÉK V (1986) Diatoms as indicators of organic pollution. Acta Hydrochim et Hydrobiol. 14 555-566.

STEVENSON RJ and PAN Y (1999) Assessing environmental conditions in rivers and streams with diatoms In: Stoermer EF and Smol JP (eds.) The Diatoms: Applications for the Environmental and Earth Sciences. Cambridge University Press, Cambridge. 11-40.

TER BRAAK CJF and VAN DAM H (1989) Inferring $\mathrm{pH}$ from diatoms: a comparison of old and new methods. Hydrobiologia 178 209-223.

TER BRAAK CJF and VERDONSCHOT PFM (1995) Canonical correspondence analysis and related multivariate methods in aquatic ecology. Aquat. Sci. 37 130-137.

VAN DAM H, MERTENS A and SINKELDAM J (1994) A coded checklist and ecological indicator values of freshwater diatoms from the Netherlands. Aquat. Ecol. 28 117-133.

WELCH EB, JACOBY JM and MAY CW (1998) Stream quality. In: Naima RJ and Bilby RE (eds.) River Ecology and Management: Lessons from the Pacific Coastal Ecoregion. Springer, New York. 69-94.

WINTER JG and DUTHIE HC (2000) Epilithic diatoms as indicators of stream total N and total P concentration. J. N. Am. Benthol. Soc. 18 32-49.

ZAMPELLA RA (1994) Characterization of surface water quality along a watershed disturbance gradient. Water Resourc. Bull. 30 605-611.

ZAMPELLA RA, LAIDIG KJ and LOWE RL (2007) Distribution of diatoms in relation to land-use and $\mathrm{pH}$ in Blackwater Coastal Plain Streams. Environ. Manage. 39 369-384.

ZAR JH (1984) Biostatistical Analysis. Prentice Hall, New Jersey. 